\title{
A Generalized Class of Exponential Type Estimators for Population Mean under Systematic Sampling Using Two Auxiliary Variables
}

\author{
Mursala Khan \\ Department of Mathematics, COMSATS Institute of Information Technology, Abbottabad 22060, Pakistan \\ Correspondence should be addressed to Mursala Khan; mursala.khan@yahoo.com
}

Received 26 June 2016; Accepted 31 July 2016

Academic Editor: Z. D. Bai

Copyright (c) 2016 Mursala Khan. This is an open access article distributed under the Creative Commons Attribution License, which permits unrestricted use, distribution, and reproduction in any medium, provided the original work is properly cited.

We have proposed a generalized class of exponential type estimators for population mean under the framework of systematic sampling using the knowledge of two auxiliary variables. The expressions for the mean square error of the proposed class of estimators have been corrected up to first order of approximation. Comparisons of the efficiency of the proposed class of estimators under the optimal conditions with the other existing estimators have been presented through a real secondary data. The statistical study provides strong evidence that the proposed class of estimators in survey estimation procedure results in substantial efficiency improvements over the other existing estimation approaches.

\section{Introduction}

In the literature of survey sampling, it is well known that the efficiencies of the estimators of the population parameters of the variable of interest can be increased by the use of auxiliary information related to auxiliary variable $x$, which is highly correlated with the variable of interest $y$. Auxiliary information may be efficiently utilized either at planning stage or at design stage to arrive at an improved estimator compared to those estimators, not utilizing auxiliary information. A simple technique of utilizing the known knowledge of the population parameters of the auxiliary variables is through ratio, product, and regression method of estimations using different probability sampling designs such as simple random sampling, stratified random sampling, cluster sampling, systematic sampling, and double sampling.

In the present paper we will use knowledge of the auxiliary variables under the framework of systematic sampling. Due to its simplicity, systematic sampling is the most commonly used probability design in survey of finite populations; see W. G. Madow and L. H. Madow [1]. Apart from its simplicity, systematic sampling provides estimators which are more efficient than simple random sampling or stratified random sampling for certain types of population; see Cochran [2], Gautschi [3], and Hajeck [4]. Later on the problem of estimating the population mean using information on auxiliary variable has also been discussed by various authors including Quenouille [5], Hansen et al. [6], Swain [7], Singh [8], Shukla [9], Srivastava and Jhajj [10], Kushwaha and Singh [11], Bahl and Tuteja [12], Banarasi et al. [13], H. P. Singh and R. Singh [14], Kadilar and Cingi [15], Koyuncu and Kadilar [16], Singh et al. [17], Singh and Solanki [18], Singh and Jatwa [19], Tailor et al. [20], Khan and Singh [21], and Khan and Abdullah [22].

Let us consider a finite population $P$ of size $N$ of distinct and identifiable units, $P_{1}, P_{2}, P_{3}, \ldots, P_{N}$ and number it from 1 to $N$ unitsin some order. A random sample of size $n$ units is selected from the first $k$ units and then every $k$ th subsequent unit is selected; thus there will be $k$ samples (clusters), each of size $n$ and observe the study variable $y$ and auxiliary variable $x$ for each and every unit selected in the sample. Let $\left(y_{i j}, x_{i j}\right)$ for $i=1,2, \ldots, k$ and $j=1,2, \ldots, n$ : denote the value of $j$ th unit in the $i$ th sample. Then the systematic sample means are defined as $\bar{y}^{*}=(1 / n) \sum_{j=1}^{n} y_{i j}$ and $\bar{x}^{*}=(1 / n) \sum_{j=1}^{n} x_{i j}$ 
are the unbiased estimators of the population means $\bar{Y}=$ $(1 / N) \sum_{j=1}^{N} y_{i j}$ and $\bar{X}=(1 / N) \sum_{j=1}^{N} x_{i j}$, respectively.

Further let

$$
\begin{aligned}
& \rho_{y}^{*}=\left\{1+(n-1) \rho_{y}\right\}, \\
& \rho_{x}^{*}=\left\{1+(n-1) \rho_{x}\right\}, \\
& \rho_{z}^{*}=\left\{1+(n-1) \rho_{z}\right\},
\end{aligned}
$$

where

$$
\begin{aligned}
& \rho_{y}=\frac{\left(y_{i j}-\bar{Y}\right)\left(y_{i j^{\prime}}-\bar{Y}\right)}{E\left(y_{i j}-\bar{Y}\right)^{2}}, \\
& \rho_{x}=\frac{\left(x_{i j}-\bar{X}\right)\left(x_{i j^{\prime}}-\bar{X}\right)}{E\left(x_{i j}-\bar{X}\right)^{2}}, \\
& \rho_{z}=\frac{\left(z_{i j}-\bar{Z}\right)\left(z_{i j^{\prime}}-\bar{Z}\right)}{E\left(z_{i j}-\bar{Z}\right)^{2}}
\end{aligned}
$$

are the corresponding intraclass correlation coefficients for the study variable $y$ and the auxiliary variables $x$ and $z$, respectively.

Similarly $\rho_{y x}=S_{y x} / S_{y} S_{x}, \rho_{y z}=S_{y z} / S_{y} S_{z}$, and $\rho_{x z}=$ $S_{x z} / S_{x} S_{z}$ are the correlation coefficients of the study and the auxiliary variables, respectively, where $S_{y}, S_{x}$, and $S_{z}$ are the population standard deviation of study variable $y$ and auxiliary variables $x$ and $z$, respectively. Also $S_{y x}, S_{y z}$, and $S_{x z}$ are the population covariances between $y$ and $x, y$ and $z$, and $z$ and $x$, respectively. Also let $C_{y}$ and $C_{x}$ and $C_{z}$ be the population coefficients of variation of the study and the auxiliary variables, respectively.

The variance of the classical estimator unbiased estimator $\bar{y}_{1}$ is given by

$$
V\left(\bar{y}_{1}\right)=\theta \bar{Y}^{2} \rho_{y}^{*} C_{y}^{2}
$$

where $\theta=((N-1) / n N)$.

Swain [7] proposed a ratio estimator in systematic sampling given by

$$
\bar{y}_{2}=\bar{y}^{*}\left(\frac{\bar{X}}{\bar{x}^{*}}\right) .
$$

The mean squared error of the above estimator is as follows:

$$
\operatorname{MSE}\left(\bar{y}_{2}\right)=\theta \bar{Y}^{2}\left[\rho_{y}^{*} C_{y}^{2}+\rho_{x}^{*} C_{x}^{2}\left(1-2 k \sqrt{\rho^{* *}}\right)\right],
$$

where $\rho^{* *}=\rho_{y}^{*} / \rho_{x}^{*}$ and $k=\rho_{y x} C_{y} / C_{x}$.

Shukla [9] suggested the following product estimator for population mean of the study variable;the suggested estimator and their mean squared error are given as follows:

$$
\begin{aligned}
\bar{y}_{3} & =\bar{y}^{*} \exp \left(\frac{\bar{z}^{*}}{\bar{Z}}\right), \\
\operatorname{MSE}\left(\bar{y}_{3}\right) & =\theta \bar{Y}^{2}\left[\rho_{y}^{*} C_{y}^{2}+\rho_{z}^{*} C_{z}^{2}\left(1+2 k^{*} \sqrt{\rho_{2}^{* *}}\right)\right],
\end{aligned}
$$

where $\rho_{2}^{* *}=\rho_{y}^{*} / \rho_{z}^{*}$ and $k^{*}=\rho_{y z} C_{y} / C_{z}$.
The usual regression estimator for population mean under systematic sampling is given as follows:

$$
\bar{y}_{4}=\bar{y}^{*}+b_{y x}\left(\bar{X}-\bar{x}^{*}\right)
$$

where $b_{y x}$ is the sample regression coefficient between $x$ and $y$.

The variance of the estimator $\bar{y}_{4}$, up to first order of approximation, is as follows:

$$
\operatorname{MSE}\left(\bar{y}_{4}\right)=\theta \rho_{y}^{*} S_{y}^{2}\left[1-\rho_{y x}^{2}\right] .
$$

Singh et al. [17] recommended ratio-product type exponential estimators and are given by

$$
\begin{aligned}
& \bar{y}_{5}=\bar{y}^{*} \exp \left(\frac{\bar{X}-\bar{x}^{*}}{\bar{X}+\bar{x}^{*}}\right), \\
& \bar{y}_{6}=\bar{y}^{*} \exp \left(\frac{\bar{x}^{*}-\bar{X}}{\bar{x}^{*}+\bar{X}}\right) .
\end{aligned}
$$

The mean square errors of the Singh et al. [17], using first order of approximation, are given as follows:

$$
\begin{aligned}
& \operatorname{MSE}\left(\bar{y}_{5}\right)=\theta \bar{Y}^{2}\left[\rho_{y}^{*} C_{y}^{2}+\frac{\rho_{x}^{*} C_{x}^{2}}{4}\left(1-4 k \sqrt{\rho^{* *}}\right)\right], \\
& \operatorname{MSE}\left(\bar{y}_{6}\right)=\theta \bar{Y}^{2}\left[\rho_{y}^{*} C_{y}^{2}+\frac{\rho_{x}^{*} C_{x}^{2}}{4}\left(1+4 k \sqrt{\rho^{* *}}\right)\right] .
\end{aligned}
$$

Tailor et al. [20] suggested a ratio-cum-product estimator for finite population mean; the recommended estimator and their first order mean square error are shown as follows:

$$
\begin{aligned}
& \bar{y}_{7}=\bar{y}^{*}\left(\frac{\bar{X}}{\bar{x}^{*}}\right)\left(\frac{\bar{z}^{*}}{\bar{Z}}\right), \\
& \operatorname{MSE}\left(\bar{y}_{7}\right)=\theta \bar{Y}^{2}\left[\rho_{y}^{*} C_{y}^{2}+\rho_{x}^{*} C_{x}^{2}\left(1-2 k \sqrt{\rho^{* *}}\right)\right. \\
& \left.\quad+\rho_{z}^{*} C_{z}^{2}\left(1-2 k^{* *} \sqrt{\rho_{1}^{* *}}\right)+2 k^{*} C_{z}^{2} \sqrt{\rho_{y}^{*} \rho_{z}^{*}}\right],
\end{aligned}
$$

where $\rho_{1}^{* *}=\rho_{x}^{*} / \rho_{z}^{*}$.

\section{The Generalized Class of Exponential Estimators}

In this section, we have proposed a generalized class of exponential type estimators for population mean of the study variable $y$, under the framework of systematic sampling as given by

$$
\begin{aligned}
& \bar{y}_{p} \\
& =\bar{y}^{*} \exp \left(\frac{f\left(\bar{X}-\bar{x}^{*}\right)}{\bar{X}+(g-1) \bar{x}^{*}}\right) \exp \left(\frac{h\left(\bar{Z}-\bar{z}^{*}\right)}{\bar{Z}+(\eta-1) \bar{z}^{*}}\right),
\end{aligned}
$$

where $-\infty<f<\infty,-\infty<h<\infty, g>0$, and $\eta>0$. 
TABLE 1: Some members of the proposed class of estimators.

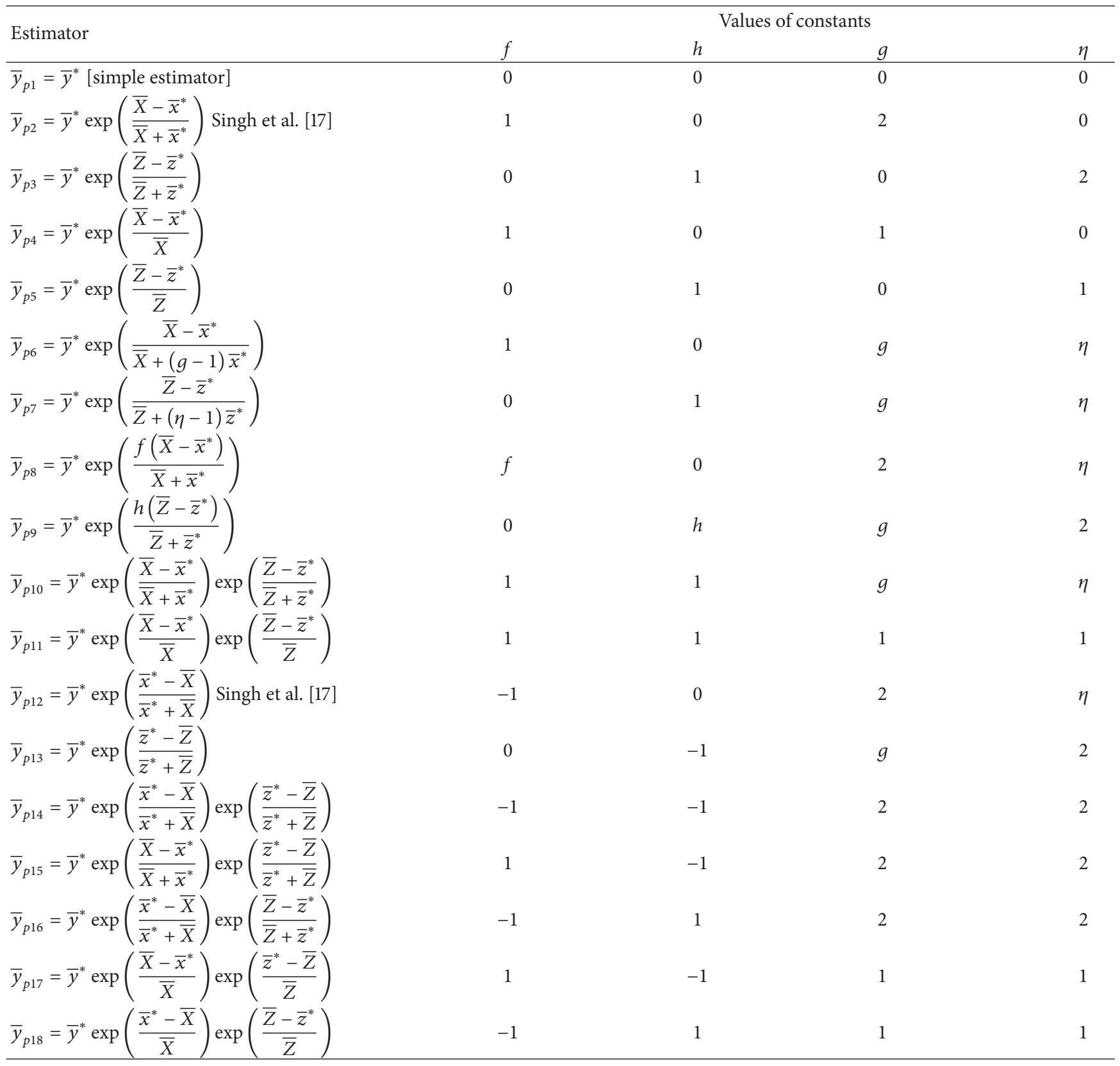

A set of some new and known members of the generalized class of exponential estimators generated from (14) for some suitable values of $f, h, g$, and $\eta$ are listed in Table 1 .

To obtain the properties of the proposed class of estimators up to first-order approximation, we use the following relative errors, symbols, and notations:

$$
\begin{aligned}
& \psi_{0}=\frac{\bar{y}^{*}-\bar{Y}}{\bar{Y}}, \\
& \psi_{1}=\frac{\bar{x}^{*}-\bar{X}}{\bar{X}}, \\
& \psi_{2}=\frac{\bar{z}^{*}-\bar{Z}}{\bar{Z}},
\end{aligned}
$$

such that

$$
E\left(\psi_{0}\right)=E\left(\psi_{1}\right)=E\left(\psi_{2}\right)=0 ;
$$

also

$$
\begin{aligned}
E\left(\psi_{0}^{2}\right) & =\theta \rho_{y}^{*} C_{y}^{2}, \\
E\left(\psi_{1}^{2}\right) & =\theta \rho_{x}^{*} C_{x}^{2}, \\
E\left(\psi_{2}^{2}\right) & =\theta \rho_{z}^{*} C_{z}^{2}, \\
E\left(\psi_{0} \psi_{1}\right) & =\theta k C_{x}^{2} \sqrt{\rho_{y}^{*} \rho_{x}^{*},}
\end{aligned}
$$




$$
\begin{aligned}
& E\left(\psi_{0} \psi_{2}\right)=\theta k^{*} C_{z}^{2} \sqrt{\rho_{y}^{*} \rho_{z}^{*}} \\
& E\left(\psi_{1} \psi_{2}\right)=\theta k^{* *} C_{z}^{2} \sqrt{\rho_{x}^{*} \rho_{z}^{*}} .
\end{aligned}
$$

Expanding (14) in terms of $\psi$ 's up to the first order of approximation, we have

$$
\begin{aligned}
\bar{y}_{p}= & \bar{Y}\left(1+\psi_{0}\right) \exp \left(\frac{-f \psi_{1}}{1+(g-1)\left(1+\psi_{1}\right)}\right) \\
& \cdot \exp \left(\frac{-h \psi_{2}}{1+(\eta-1)\left(1+\psi_{2}\right)}\right) .
\end{aligned}
$$

Further simplify

$$
\begin{gathered}
\bar{y}_{p}-\bar{Y}=\bar{Y}\left[\psi_{0}-\frac{f}{g} \psi_{1}-\frac{h}{\eta} \psi_{2}-\frac{f}{g} \psi_{0} \psi_{1}-\frac{h}{\eta} \psi_{0} \psi_{2}\right. \\
\left.+\delta_{1} \psi_{1}^{2}+\delta_{2} \psi_{2}^{2}+\delta_{3} \psi_{1} \psi_{2}\right],
\end{gathered}
$$

where $\delta_{1}=f / g-f / g^{2}+f^{2} / 2 g^{2}, \delta_{2}=h / \eta-h / \eta^{2}+h^{2} / 2 \eta^{2}$, and $\delta_{3}=f h / g \eta$.

On squaring and taking expectation on both sides of (19), we get the mean square error of $\bar{y}_{p}$, up to the first degree of approximation, as

$$
\begin{aligned}
& \operatorname{MSE}\left(\bar{y}_{p}\right)=\theta \bar{Y}^{2}\left[\rho_{y}^{*} C_{y}^{2}+\lambda_{1}^{2} \rho_{x}^{*} C_{x}^{2}+\lambda_{2}^{2} \rho_{z}^{*} C_{z}^{2}\right. \\
& -2 \lambda_{1} k C_{x}^{2} \sqrt{\rho_{y}^{*} \rho_{x}^{*}}-2 \lambda_{2} k^{*} C_{z}^{2} \sqrt{\rho_{y}^{*} \rho_{z}^{*}} \\
& \left.+2 \lambda_{1} \lambda_{2} k^{* *} C_{z}^{2} \sqrt{\rho_{x}^{*} \rho_{z}^{*}}\right],
\end{aligned}
$$

where $\lambda_{1}=f / g$ and $\lambda_{2}=h / \eta$.

By partially differentiating (20) with respect to $\lambda_{1}$ and $\lambda_{2}$, we get the optimum value of $\lambda_{1}$ and $\lambda_{2}$ as given by

$$
\begin{aligned}
& \lambda_{1}=\frac{\delta_{1} \sqrt{\rho^{* *}}}{\delta_{2}}, \\
& \lambda_{2}=\frac{\delta_{3} C_{x}^{2} \sqrt{\rho_{2}^{* *}}}{\delta_{2}},
\end{aligned}
$$

where $\delta_{1}=k C_{x}^{2}-k^{*} k^{* *} C_{z}^{2}, \delta_{2}=C_{x}^{2}-k^{2 * *} C_{z}^{2}$, and $\delta_{3}=$ $k^{*}-k k^{* *}$.

Substituting the optimal values of $\lambda_{1}$ and $\lambda_{2}$ in (20) we obtain the minimum mean square error of the estimator $\bar{y}_{m}$ as follows:

$$
\begin{aligned}
& \operatorname{MSE}\left(\bar{y}_{p}\right)_{\min }=\theta \bar{Y}^{2} \rho_{y}^{*}\left[C_{y}^{2}\right. \\
& +\frac{C_{x}^{2}}{\delta_{2}^{2}}\left\{\left(\delta_{1}^{2}+\delta_{3}^{2} C_{x}^{2} C_{z}^{2}+2 k^{* *} C_{z}^{2} \delta_{1} \delta_{3}\right)\right. \\
& \left.\left.-2 \delta_{2}\left(k \delta_{1}+k^{*} C_{z}^{2} \delta_{3}\right)\right\}\right] .
\end{aligned}
$$

\section{Comparison of Efficiency}

In this section, we have found some theoretical efficiencies conditions under which the proposed estimator performs better than the other relevant existing estimators by comparing the generalized class of exponential type estimators with other existing estimators.

(i) By (22) and (3), $\operatorname{MSE}\left(\bar{y}_{p}\right) \leq \operatorname{MSE}\left(\bar{y}_{1}\right)$ if

$$
\begin{aligned}
& {\left[2 \delta_{1} \delta_{2} k+2 \delta_{2} \delta_{3} k^{*} C_{z}^{2}-\delta_{1}^{2}-\delta_{3}^{2} C_{x}^{2} C_{z}^{2}-2 k^{* *} C_{z}^{2} \delta_{1} \delta_{3}\right]} \\
& \quad \geq 0 .
\end{aligned}
$$

(ii) $\operatorname{By}(22)$ and (5), $\operatorname{MSE}\left(\bar{y}_{p}\right) \leq \operatorname{MSE}\left(\bar{y}_{2}\right)$ if

$$
\begin{aligned}
& {\left[\delta_{2}^{2} \rho_{x}^{*}\left(1-2 k \sqrt{\rho^{* *}}\right)-\rho_{y}^{*}\left(\delta_{1}^{2}+\delta_{3}^{2} C_{x}^{2} C_{z}^{2}\right.\right.} \\
& \left.\left.\quad+2 \delta_{1} \delta_{3} k^{* *} C_{z}^{2}-2 \delta_{1} \delta_{2} k-2 \delta_{2} \delta_{3} k^{*} C_{z}^{2}\right)\right] \geq 0 .
\end{aligned}
$$

(iii) By (22) and (7), $\operatorname{MSE}\left(\bar{y}_{p}\right) \leq \operatorname{MSE}\left(\bar{y}_{3}\right)$ if

$$
\begin{gathered}
{\left[\rho_{z}^{*} C_{z}^{2} \delta_{2}^{2}\left(1+2 k^{*} \sqrt{\rho_{2}^{* *}}\right)-\rho_{y}^{*} C_{x}^{2}\left(\delta_{1}^{2}+\delta_{3}^{2} C_{x}^{2} C_{z}^{2}\right.\right.} \\
\left.\left.+2 k^{* *} C_{z}^{2} \delta_{1} \delta_{3}-2 k \delta_{1} \delta_{2}-2 \delta_{2} \delta_{3} k^{*} C_{z}^{2}\right)\right] \geq 0
\end{gathered}
$$

(iv) By (22) and (9), $\operatorname{MSE}\left(\bar{y}_{p}\right) \leq \operatorname{MSE}\left(\bar{y}_{4}\right)$ if

$$
\begin{gathered}
{\left[C _ { x } ^ { 2 } \left(2 k \delta_{1} \delta_{2}+2 \delta_{2} \delta_{3} k^{*} C_{z}^{2}-\delta_{1}^{2}-\delta_{3}^{2} C_{x}^{2} C_{z}^{2}\right.\right.} \\
\left.\left.-2 k^{* *} C_{z}^{2} \delta_{1} \delta_{3}\right)-\rho_{y x}^{2} \delta_{2}^{2} C_{y}^{2}\right] \geq 0
\end{gathered}
$$

(v) By (22) and (11), $\operatorname{MSE}\left(\bar{y}_{p}\right) \leq \operatorname{MSE}\left(\bar{y}_{5}\right)$ if

$$
\begin{aligned}
& {\left[\frac{\rho_{x}^{*}}{4}\left(1-4 k \sqrt{\rho^{* *}}\right)-\frac{\rho_{y}^{*}}{\delta_{2}^{2}}\left(\delta_{1}^{2}+\delta_{3}^{2} C_{x}^{2} C_{z}^{2}\right.\right.} \\
& \left.\left.\quad+2 k^{* *} C_{z}^{2} \delta_{1} \delta_{3}-2 k \delta_{1} \delta_{2}-2 \delta_{2} \delta_{3} k^{*} C_{z}^{2}\right)\right] \geq 0 .
\end{aligned}
$$

(vi) By (22) and (12), $\operatorname{MSE}\left(\bar{y}_{p}\right) \leq \operatorname{MSE}\left(\bar{y}_{6}\right)$ if

$$
\begin{aligned}
& {\left[\frac{\rho_{x}^{*}}{4}\left(1+4 k \sqrt{\rho^{* *}}\right)-\frac{\rho_{y}^{*}}{\delta_{2}^{2}}\left(\delta_{1}^{2}+\delta_{3}^{2} C_{x}^{2} C_{z}^{2}\right.\right.} \\
& \left.\left.\quad+2 k^{* *} C_{z}^{2} \delta_{1} \delta_{3}-2 k \delta_{1} \delta_{2}-2 \delta_{2} \delta_{3} k^{*} C_{z}^{2}\right)\right] \geq 0 .
\end{aligned}
$$

(vii) By (22) and (13), $\operatorname{MSE}\left(\bar{y}_{p}\right) \leq \operatorname{MSE}\left(\bar{y}_{7}\right)$ if 
TABLE 2: The mean square errors (MSEs) of the estimators and the percent relative efficiencies (PREs) with respect to $\bar{y}_{1}$.

\begin{tabular}{lcc}
\hline & Population & PRE $\left(\bar{y}_{*}, \bar{y}_{1}\right)$ \\
\hline Estimator & MSE $\left(\bar{y}_{*}\right)$ & 100.00 \\
$\bar{y}_{1}$ & 1455.08 & 389.62 \\
$\bar{y}_{2}$ & 373.32 & 189.45 \\
$\bar{y}_{3}$ & 768.06 & 3326.66 \\
$\bar{y}_{4}$ & 43.74 & 177.43 \\
$\bar{y}_{5}$ & 820.09 & 139.32 \\
$\bar{y}_{6}$ & 1044.42 & 777.79 \\
$\bar{y}_{7}$ & 187.08 & 6158.08 \\
$\bar{y}_{p}$ & 23.63 & \\
\hline
\end{tabular}

$$
\rho_{y}^{*} \leq \frac{\delta_{2}^{2}\left[\rho_{x}^{*} C_{x}^{2}\left(1-2 k \sqrt{\rho^{* *}}\right)+\rho_{z}^{*} C_{z}^{2}\left(1-2 k^{* *} \sqrt{\rho_{1}^{* *}}\right)+2 k^{*} C_{z}^{2} \sqrt{\rho_{y}^{*} \rho_{z}^{*}}\right]}{C_{x}^{2}\left(\delta_{1}^{2}+\delta_{3}^{2} C_{x}^{2} C_{z}^{2}+2 k^{* *} C_{z}^{2} \delta_{1} \delta_{3}-2 k \delta_{1} \delta_{2}-2 \delta_{2} \delta_{3} k^{*} C_{z}^{2}\right)} .
$$

\section{Empirical Study}

To examine the merits of the proposed estimator over the other existing estimators at optimum conditions, we have considered natural population data sets from the literature. The sources of population are given as follows.

Population (Source: Tailor et al. [20]). Consider

$$
\begin{aligned}
N & =15, \\
n & =3, \\
\bar{X} & =44.47, \\
\bar{Y} & =80, \\
\bar{Z} & =48.40, \\
C_{y} & =0.56, \\
C_{x} & =0.28, \\
C_{z} & =0.43, \\
S_{y}^{2} & =2000, \\
S_{x}^{2} & =149.55, \\
S_{z}^{2} & =427.83, \\
S_{y x} & =538.57, \\
S_{y z} & =-902.86, \\
S_{x z} & =-241.06, \\
\rho_{y x} & =0.9848, \\
\rho_{y z} & =-0.9760,
\end{aligned}
$$

$$
\begin{aligned}
\rho_{x z} & =-0.9530, \\
\rho_{y} & =0.6652, \\
\rho_{x} & =0.707, \\
\rho_{z} & =0.5487 .
\end{aligned}
$$

The percent relative efficiencies (PREs) of the stated estimators with respect to the usual unbiased estimator are obtained from the following mathematical formula:

$$
\operatorname{PRE}\left(\bar{y}_{*}, \bar{y}_{1}\right)=\frac{\operatorname{MSE}\left(\bar{y}_{1}\right)}{\operatorname{MSE}\left(\bar{y}_{*}\right)} \times 100
$$

where $*=1,2,3,4,5,6,7$, and $p$.

\section{Conclusion}

In this paper we proposed a generalized class of exponential type estimators for the population mean of study variable $y$, when information is available on two auxiliary variables under the framework of systematic sampling scheme. The properties of the proposed estimator are derived up to first order of approximation. The proposed estimator is compared with other present estimators, both as theoretical and empirical efficiency comparisons. We have also judged the performance of the proposed estimator for a known natural population dataset; see Tailor et al. [20]. Results are given in Table 2 which shows that performances of the proposed generalized class of exponential type estimator are more efficient than the other existing estimators by smaller mean square errors and the higher percent relative efficiencies of the estimators. Hence it is preferable to use the proposed estimator in practical surveys. 


\section{Competing Interests}

The author declares that there is no conflict of interests regarding the publication of this paper.

\section{References}

[1] W. G. Madow and L. H. Madow, "On the theory of systematic sampling. I," Annals of Mathematical Statistics, vol. 15, pp. 1-24, 1944.

[2] W. G. Cochran, "Relative accuracy of systematic and stratified random samples for a certain class of populations," The Annals of Mathematical Statistics, vol. 17, pp. 164-177, 1946.

[3] W. Gautschi, "Some remarks on systematic sampling," Annals of Mathematical Statistics, vol. 28, pp. 385-394, 1957.

[4] J. Hajeck, "Optimum strategy and other problems in probability sampling," Casopis pro Pestovani Matematiky, vol. 84, pp. 387423, 1959.

[5] M. H. Quenouille, "Notes on bias in estimation," Biometrika, vol. 43, pp. 353-360, 1956.

[6] M. H. Hansen, W. N. Hurwitz, and M. Gurney, "Problems and methods of the sample survey of business," Journal of the American Statistical Association, vol. 41, no. 234, pp. 173-189, 1946.

[7] A. K. P. C. Swain, "The use of systematic sampling ratio estimate," Journal of the Indian Statistical Association, vol. 2, pp. 160-164, 1964.

[8] M. P. Singh, "Ratio-cum-product method of estimation," Metrika, vol. 12, pp. 34-42, 1967.

[9] N. D. Shukla, "Systematic sampling and product method of estimation," in Proceeding of the all India Seminar on Demography and Statistics, Varanasi, India, 1971.

[10] S. K. Srivastava and H. S. Jhajj, "A class of estimators of the population mean using multi-auxiliary information," Calcutta Statistical Association Bulletin, vol. 32, no. 125-126, pp. 47-56, 1983.

[11] K. S. Kushwaha and H. P. Singh, "Class of almost unbiased ratio and product estimators in systematic sampling," Journal of the Indian Society of Agricultural Statistics, vol. 41, no. 2, pp. 193205, vi, 1989.

[12] S. Bahl and R. K. Tuteja, "Ratio and product type exponential estimators," Journal of Information \& Optimization Sciences, vol. 12, no. 1, pp. 159-164, 1991.

[13] Banarasi, S. N. S. Kushwaha, and K. S. Kushwaha, "A class of ratio, product and difference (R.P.D.) estimators in systematic sampling," Microelectronics Reliability, vol. 33, no. 4, pp. 455457, 1993.

[14] H. P. Singh and R. Singh, "Almost unbiased ratio and product type estimators in systematic sampling," Questiio, vol. 22, no. 3, pp. 403-416, 1998.

[15] C. Kadilar, and H. Cingi, "An improvement in estimating the population mean by using the correlation coefficient," Hacettepe Journal of Mathematics and Statistics, vol. 35, no. 1, pp. 103-109, 2006.

[16] N. Koyuncu and C. Kadilar, "Family of estimators of population mean using two auxiliary variables in stratified random sampling," Communications in Statistics: Theory and Methods, vol. 38, no. 13-15, pp. 2398-2417, 2009.

[17] H. P. Singh, R. Tailor, and N. K. Jatwa, "Modified ratio and product estimators for population mean in systematic sampling,"
Journal of Modern Applied Statistical Methods, vol. 10, no. 2, pp. 424-435, 2011.

[18] H. P. Singh and R. S. Solanki, "An efficient class of estimators for the population mean using auxiliary information in systematic sampling," Journal of Statistical Theory and Practice, vol. 6, no. 2, pp. 274-285, 2012.

[19] H. P. Singh and N. K. Jatwa, "A class of exponential type estimators in systematic sampling," Economic Quality Control, vol. 27, no. 2, pp. 195-208, 2012.

[20] T. Tailor, N. Jatwa, and H. P. Singh, "A ratio-cum-product estimator of finite population mean in systematic sampling," Statistics in Transition, vol. 14, no. 3, pp. 391-398, 2013.

[21] M. Khan and R. Singh, "Estimation of population mean in chain ratio-type estimator under systematic sampling," Journal of Probability and Statistics, vol. 2015, Article ID 248374, 2 pages, 2015.

[22] M. Khan and H. Abdullah, "A note on a differencetype estimator for population mean under twophase sampling design," SpringerPlus, vol. 5, article 723, 7 pages, 2016. 


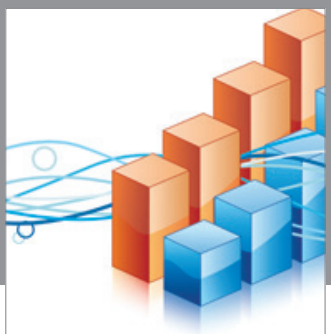

Advances in

Operations Research

vatem alat4



\section{The Scientific} World Journal

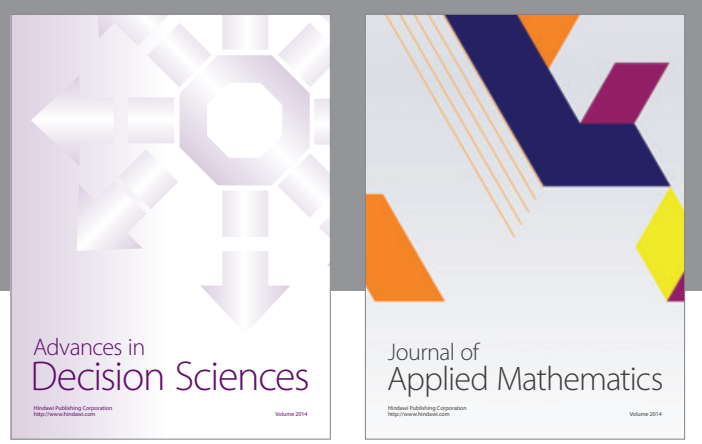

Algebra

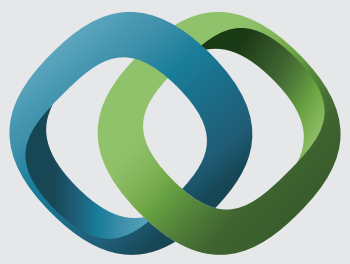

\section{Hindawi}

Submit your manuscripts at

http://www.hindawi.com
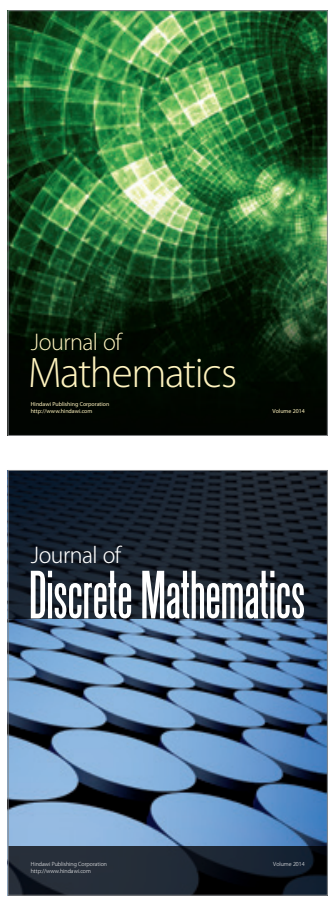



Mathematical Problems in Engineering
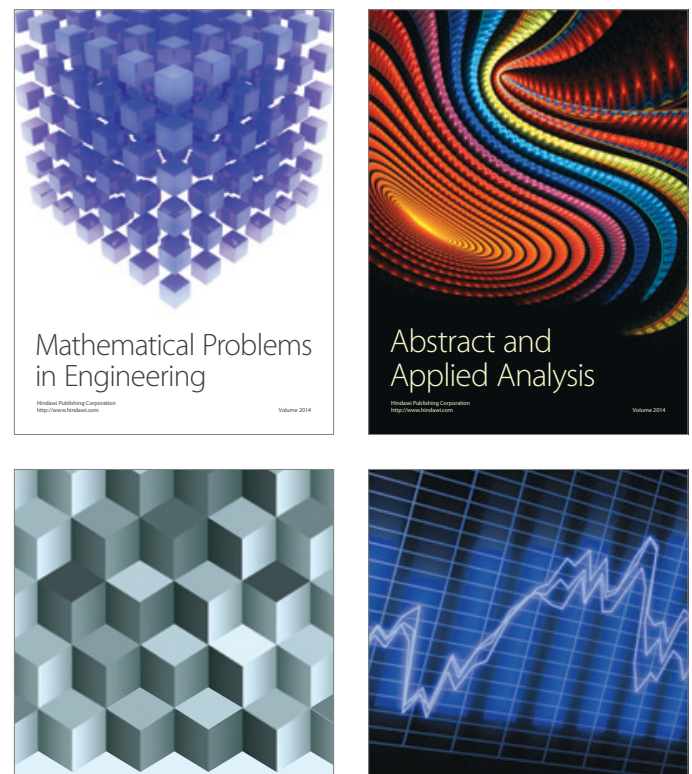

Journal of

Function Spaces

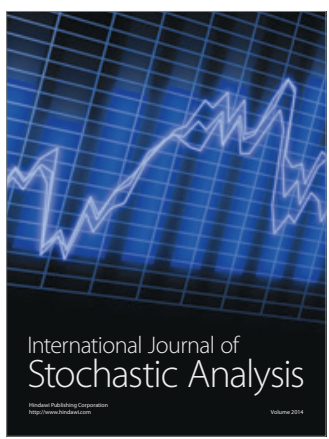

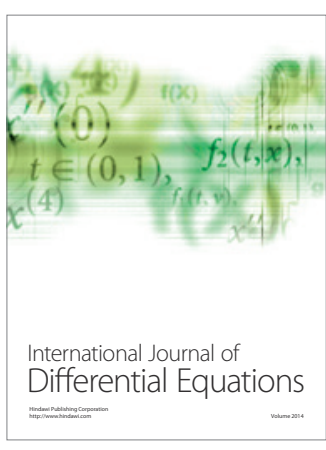
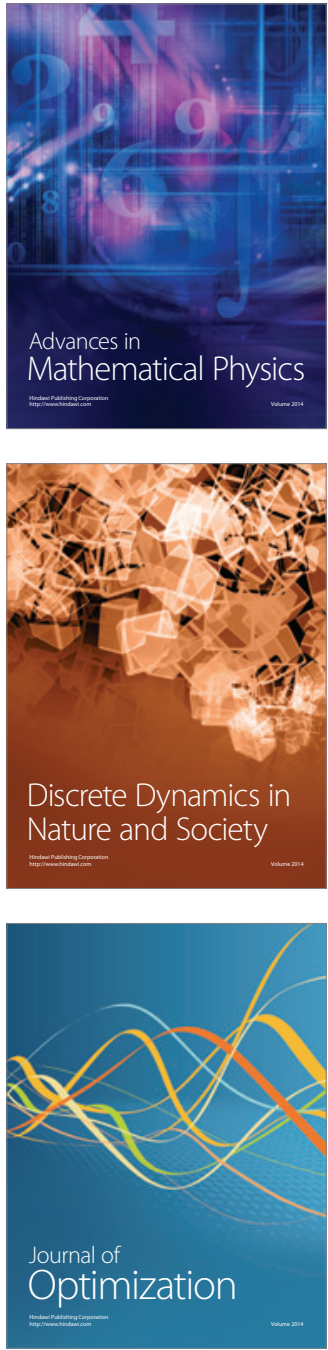